\title{
Correlação entre os resultados obtidos em testes motores e o desempenho em exergames em idosos
}

\author{
Correlation between results obtained in motor tests and \\ performance in exergames in elderly
}

\author{
Mateus Pelisser, ${ }^{1}$ Daniel Carlos Garlipp, ${ }^{2}$ Marcelo Morganti Sant'Anna' \\ 'Faculdade Cenecista de Osório (FACOS/RS), Osório, RS, Brasil. \\ ZUniversidade Luterana do Brasil (ULBRA/RS), Porto Alegre, RS, Brasil.
}

Recebido em: 11/01/2017 / Aceito em: 24/05/2017 / Publicado em: 30/06/2017 dcgarlipp@gmail.com

\section{RESUMO}

Objetivo: correlacionar resultados obtidos em testes motores realizados em idosos com o seu desempenho em exergames. Método: a amostra foi composta por 34 idosos, divididos em 4 grupos: grupo homens treinados ( $\mathrm{n}=8$ ), grupo homens não treinados ( $\mathrm{n}=$ 9), grupo mulheres treinadas $(n=8)$ e grupo mulheres não treinadas ( $\mathrm{n}=9$ ). Para avaliação do desempenho motor utilizou-se o exergame Your Shape Fitness Evolved $2012^{\circledR}$ do XBOX $360^{\circledR}$, o teste Sentar e Alcançar, a Escala de Equilíbrio de Berg e o teste de levantar da cadeira em 30 segundos. Para verificar possíveis diferenças estatisticamente significativas entre os grupos treinado e não treinado foi utilizado o teste t para amostras independentes, para cada sexo, em cada variável utilizada (flexibilidade, equilíbrio, força de membros inferiores e exergame). Para verificar correlação entre os escores obtidos no exergame e os resultados nos testes motores, foi utilizada a correlação de Spearman. Para as análises estatísticas, utilizou-se o programa estatístico SPSS for Windows 20.0, com nível de significância de 5\%. Resultados: foi identificada correlação significativa entre os escores do Exergame e os testes motores de flexibilidade e força de membros inferiores em ambos os sexos. No grupo masculino, a correlação da flexibilidade e exergame foi de $r=0,802(p=0,000)$ e da força de membros inferiores e exergame foi de $r=0,833$ ( $p=$ $0,000)$. No grupo feminino, a correlação da flexibilidade e exergame foi de $r=0,641(p=0,006)$ e da força de membros inferiores e exergame foi de $r=0,898$ ( $p=$ $0,000)$. Na comparação das variáveis equilíbrio, flexibilidade, força de membros inferiores e escore do exer- game entre grupos treinados e não-treinados, divididos por sexo, os grupos treinados apresentaram maiores valores em todos os parâmetros. Considerações finais: considera-se que o exergame utilizado no presente estudo pode servir como instrumento de avaliação da capacidade motora de idosos. Ainda, idosos treinados apresentam melhor desempenho motor em testes específicos e escores do exergame quando comparados a idosos não treinados.

Palavras-chave: Desempenho Motor; Idoso; Testes de Aptidão; Exergame.

\section{ABSTRACT}

Objective: to correlate results obtained in motor tests performed in the elderly with their performance in exergames. Method: the sample consisted of 34 elderly people, divided into 4 groups: trained men $(n=$ 8), untrained men ( $n=9)$, trained women $(n=8)$ and untrained women $(n=9)$. For motor performance evaluation the $\mathrm{XBOX} 360^{\circledR}$ exergame Your Shape Fitness Evolved 2012 ${ }^{\circledR}$, the Sit and Go test, the Berg Balance Scale and the chair lift test in 30 seconds were used. In order to verify possible statistically significant differences between the trained and untrained groups, the t test for independent samples was used, for each sex, in each variable used (flexibility, balance, lower limb strength and exergame). In order to verify correlation between exergame scores and motor test results the Spearman correlation test was used. Statistical analysis was performed using the statistical software SPSS for Windows 20.0, with a significance level of 5\%. Results: 
significant correlation was found between the Exergame scores and the motor tests of flexibility and lower limb strength in both genders. In the male group the correlation of flexibility and exergame was $r=0.802$ $(p=0.000)$ and limb strength and exergame was $r=$ $0.833(p=0.000)$. In the female group the correlation of flexibility and exergame was $r=0.641(p=$ $0.006)$ and strength of lower limbs and exergame was $r=0.898(p=0.000)$. In the comparison of variables balance, flexibility, lower limb strength and exergame score between trained and untrained groups, divided by gender, the trained groups showed higher values in all parameters. Closing remarks: it is considered that the exergame used in the present study can serve as a tool to evaluate the motor capacity of the elderly. Still, trained elderly individuals present better motor performance in specific tests and exergame scores when compared to untrained elderly.

Keywords: Motor Performance; Aged; Aptitude Tests; Exergame.

\section{INTRODUÇ̃̃O}

O processo de envelhecimento, aliado à vida sedentária, é um dos principais fatores de complicações na saúde de inúmeros idosos. De acordo com Davini e Nunes, ${ }^{1}$ a diminuição da força muscular e subsequente comprometimento da função motora, associados ao processo de envelhecimento, afetam diretamente a vida desses indivíduos, diminuindo suas habilidades em tarefas simples como caminhar, dificultando a realização de atividades de vida diária, comprometendo a qualidade de vida e a saúde mental dessa população.

Benefícios obtidos com a prática rotineira de exercícios físicos são numerosos para essa população, destacando-se a diminuição de dores nas articulações, a manutenção da força e flexibilidade, o retardamento da perda da capacidade funcional, a melhoria do perfil lipídico, a melhora da capacidade cognitiva, dentre outras. ${ }^{2-4}$ Para Civinski ${ }^{5}$ idosos que não realizam exercícios físicos estão mais vulneráveis aos acidentes do dia a dia, pelo fato de não terem mais o equilíbrio necessário, sua força não corresponder às necessidades, e a resistência não permitir que se execute qualquer movimento acima da sua condição.

Nesse sentido, para além da recomendação de realização de atividades físicas periódicas, vem surgindo, nos últimos anos, métodos alternativos que podem ser aliados à manutenção da saúde da população em geral. Dentre eles, estão os exergames (mistura de videogame interativo com exercício físico), que possibilitam a interação física dos jogadores com os ambientes virtuais. Sua utilização já se destaca na área da reabilitação, pois são capazes de promover o movimento das partes superiores e inferiores do corpo como forma de exercício, além de proporcionar uma percepção em uma realidade estrutural diferente. ${ }^{6}$ Com a utilização dos exergames, os praticantes podem realizar movimentos e gestos corporais sem a necessidade de controles, sendo que diversos autores tratam de sua eficácia, para melhora do desempenho motor. ${ }^{7-9}$

Estudos como o de Dos Santos e Magalhães, ${ }^{9}$ demonstram a eficácia dos exergames em relação à melhora da aptidão física. De acordo com estes autores, a conduta com o videogame melhora significativamente a capacidade funcional, podendo ser utilizada no âmbito de prevenção, promoção e até mesmo no tratamento de diversas doenças, como obesidade e doenças cardíacas, além de serem altamente motivadoras. Meneghini et al. ${ }^{10}$ descrevem que os exergames podem contribuir não apenas para o aumento do consumo calórico, mas para diminuição do sedentarismo, benefícios psicológicos e de interação social, resultando num ganho fisiológico geral do organismo do indivíduo. Vieira et al. Destacam como aspecto positivo o envolvimento com a atividade e a motivação proporcionada pelos exergames, propondo a vivência de novos esportes e a diminuição do sedentarismo. ${ }^{11}$

A conduta com esta ferramenta também tem exercido papel importante na melhora dos níveis de desempenho motor de idosos, relacionado a qualidades, como equilíbrio, flexibilidade e força de membros inferiores, além de promover melhora da capacidade funcional, cognitiva e até em questões emocionais dos idosos. . $^{6,12-15}$

A prática do exergame como promoção de exercício para idosos parece já ser uma realidade, além de mostrar-se efetiva na melhoria da condição física dos idosos. Sendo assim, considerando tais achados acredita-se que o seu uso também possa servir como avaliação da capacidade motora do idoso, já que o jogo apresenta escores de desempenho durante a execução do jogo. Assim, correlacionar os resultados do exergame com os testes motores para idosos, torna-se importante para identificar a aplicabilidade do exergame também enquanto instrumento de avaliação. Sendo assim, o objetivo do presente estudo foi correlacionar resultados de testes motores realizados em idosos com o seu desempenho em exergames.

\section{METODOLOGIA}

A amostra foi composta por 34 idosos divididos em quatro grupos (homens treinados: $n=8$; homens não treinados: $n=9$; mulheres treinadas: $n=8$; mulheres não treinadas: $n=9$ ), com idade a partir de 60 anos, residentes do litoral norte do Rio Grande do Sul. Os homens e as mulheres treinadas participavam de um projeto social de atividade física do município de Maquiné/RS. As atividades são orientadas por profissionais de Educação Física e realizadas 2 vezes por semana, com duração de 1 hora/sessão. As atividades realizadas são de dança, caminhada orientada e ginástica localizada. O grupo de não treinados foram recrutados também no município de Maquiné/RS. Todos os participantes foram convidados e fizeram parte da amostra a partir da aprovação e assinatura do Termo de Consentimento Livre e Esclarecido (TCLE). Para confirmação do nível de atividade física dos treinados e não treinados, utilizou-se a versão curta do Questionário Internacional de Atividade Física (IPAQ) proposto por Benedetti et al. ${ }^{16}$

Os critérios de inclusão da amostra foram: ter idade a partir de 60 anos e possuir condições de realizar os testes de desempenho motor específico e no exergame. Como critérios de exclusão estabeleceu-se a queixa de desconforto/dor ou anomalia antes ou durante a realização dos testes sugeridos no presente estudo. 
Para além da medição de variáveis morfológicas de estatura, peso e cálculo do índice de massa corporal (IMC), os idosos foram submetidos a testes de desempenho motor e ao exergame. Para o teste e exergame, foi utilizado o console $\mathrm{XBOX} 360^{\circledR}$, aliado ao sensor de movimentos KINECT $^{\circledR}$. O exergame utilizado foi o jogo Your Shape Fitnnes Evolved $2012^{\circledR}$, desenvolvido pela empresa UBISOFT ${ }^{\circledR}$. Para realização do teste com este jogo, que é dividido em diversas modalidades, foi considerado o teste de "yoga", parte integrante do jogo, que envolve movimentos básicos que utilizam valências de flexibilidade, força de membros inferiores e equilíbrio, compatíveis aos testes motores alvos do estudo. Os idosos que realizaram o teste com exergame foram previamente orientados sobre sua execução, bem como puderam solicitar o encerramento da realização do mesmo em qualquer momento. O teste com exergame comtemplou oito minutos e os idosos tiveram direito a três intervalos: o primeiro aos dois minutos de atividade; o segundo aos quatro minutos de atividade e o terceiro aos seis minutos de atividade. Caso o participante não solicitasse os intervalos, a conduta com o exergame continuaria até o fechamento dos oito minutos estipulados. Ao final do teste com exergame, a própria ferramenta calculou a porcentagem de acertos de movimentos do indivíduo, em uma escala de 0 a $100 \%$. Para níveis mais detalhados de score de pontuação, foi tomado nota da pontuação referente à cada acerto de movimento realizado.

Para os testes de desempenho motor específicos, foram consideradas três valências físicas: equilíbrio, flexibilidade e força de membros inferiores. Em relação ao teste de equilíbrio, foi utilizada a Versão Brasileira da Escala de Equilíbrio de Berg. ${ }^{17}$ Trata-se de uma avaliação funcional, baseada em 14 itens comuns do dia a dia, que, de acordo com sua execução, são diferentemente pontuados, em uma escala de zero (não houve execução) à quatro (executou perfeitamente). Ao final do teste, o score final decretaria o nível de equilíbrio do indivíduo, da seguinte maneira: entre 41 e 56 pontos, baixo risco de queda; entre 21 e 40 pontos, médio risco de queda; e entre zero e 20 pontos, elevado risco de queda.

Para o teste de flexibilidade, foi utilizado o teste de sentar e alcançar, proposto por Wells e Dillon, ${ }^{18}$ anotando-se o melhor resultado, após 2 tentativas. Já, para o teste de força de membros inferiores, foi utilizado o teste de Levantar da Cadeira em 30 segundos, proposto por Matsudo, ${ }^{18}$ sendo o resultado determinado através da contagem da quantidade de vezes que o participante executou corretamente os movimentos de sentar e levantar da cadeira.

Para a estatística descritiva foram utilizados os valores da média e do desvio-padrão. Quanto à estatística inferencial, para verificar possíveis diferenças estatisticamente significativas entre os grupos treinado e não treinado, foi utilizado o teste $t$ para amostras independentes, em cada sexo, em cada variável analisada. Para verificar possíveis correlações significativas entre os escores obtidos no exergame e os resultados nos testes motores, foi utilizada a correlação de Spearman. Quanto aos valores de $r$ foram seguidas as seguintes normativas: se $r<0,3=$ correlação fraca; $0,3>r<0,6$ $=$ correlação moderada; $r>0,6=$ correlação forte. Para todas as análises foi utilizado o programa estatístico SPSS for Windows versão 20.0. O nível de significância adotado foi de $5 \%$.

O presente estudo teve a aprovação do Comitê de Ética em Pesquisa da FACOS, sob o número 49295215.8.0000.5591.

\section{RESULTADOS}

Na tabela 1 estão descritos os valores da média e desvio-padrão da idade, peso, estatura, índice de massa corporal (IMC) e valores obtidos no teste IPAQ.

Em relação à variável idade, a mesma variou entre 60 e 77 anos, sendo que o grupo das mulheres não treinadas apresentou maior média. Na variável peso, o grupo que apresentou maior média foi o de homens não treinados $(74,33 \mathrm{~kg})$, enquanto que o grupo de mulheres não treinadas apresentou a menor média $(69,78 \mathrm{~kg})$. Em relação à variável estatura, os homens de ambos os grupos apresentaram média semelhante $(1,68 \mathrm{~m})$, enquanto que as mulheres não treinadas $(1,61 \mathrm{~m})$ são ligeiramente mais altas do que o grupo de mulheres treinadas $(1,60 \mathrm{~m})$. Quanto ao IMC, os homens de ambos os grupos apresentaram média semelhante, enquanto que as mulheres treinadas $\left(28,42 \mathrm{~kg} / \mathrm{m}^{2}\right)$ apresentaram média superior ao grupo de mulheres não treinadas $\left(26,88 \mathrm{~kg} / \mathrm{m}^{2}\right)$.

De acordo com o questionário IPAQ, idosos que apresentam 150 minutos ou mais de atividade física por semana ( $\mathrm{min} / \mathrm{sem}$ ) são considerados fisicamente ativos, e os que apresentam valores menores que 150 minutos de atividade física por semana são considerados fisicamente inativos. ${ }^{16}$ Sendo assim, considerando os minutos de atividade física por semana dos grupos de homens treinados $(156,88 \mathrm{~min} / \mathrm{sem})$, homens não treinados $(105,00 \mathrm{~min} / \mathrm{sem})$, mulheres treinadas $(168,13$ $\mathrm{min} / \mathrm{sem})$ e mulheres não treinadas $(111,67 \mathrm{~min} / \mathrm{sem})$, constata-se que os grupos de idosos treinados são classificados como fisicamente ativos e os grupos de idosos não treinados classificados como fisicamente inativos.

Na tabela 2 é possível observar que os homens do grupo treinado superam o grupo não treinado de forma significativa nos testes motores de flexibilidade e força de membros inferiores, além do escore no Exergame. Porém, não há diferença significativa entre os níveis de equilíbrio encontrados entre os grupos.

Tabela 1 - Descrição da Amostra.

\begin{tabular}{|c|c|c|c|c|}
\hline Variável & Homens treinados & Homens não treinados & Mulheres treinadas & Mulheres não treinadas \\
\hline Idade (anos) & $64,75 \pm 3,94$ & $65,67 \pm 3,41$ & $66,25 \pm 4,56$ & $66,56 \pm 5,41$ \\
\hline Peso (kg) & $73,50 \pm 10,75$ & $74,33 \pm 4,07$ & $72,13 \pm 6,66$ & $69,78 \pm 5,36$ \\
\hline Estatura (m) & $1,68 \pm 0,06$ & $1,68 \pm 0,04$ & $160 \pm 0,04$ & $1,61 \pm 0,05$ \\
\hline IMC $\left(\mathrm{kg} / \mathrm{m}^{2}\right)$ & $26,12 \pm 2,97$ & $26,46 \pm 1,57$ & $28,42 \pm 3,73$ & $26,88 \pm 2,33$ \\
\hline IPAQ (min/sem) & $156,88 \pm 9,84$ & $105 \pm 17,78$ & $168,13 \pm 14,38$ & $111,67 \pm 19,26$ \\
\hline
\end{tabular}


Tabela 2 - Comparação entre os escores das variáveis analisadas entre os grupos treinado e não treinado para o sexo masculino.

\begin{tabular}{lcccc}
\hline Variável & $\begin{array}{c}\text { Homens Treinados } \\
\text { Média } \pm \text { DP }\end{array}$ & $\begin{array}{c}\text { Homens não treinados } \\
\text { Média } \pm \text { DP }\end{array}$ & $\boldsymbol{t}$ & Sig. \\
\hline Flexibilidade & $29,71 \pm 6,86$ & $19,55 \pm 6,80$ & 3,060 & 0,008 \\
Equilíbrio & $52,25 \pm 2,76$ & $50,56 \pm 1,42$ & 1,559 & 0,149 \\
Força de membros inferiores & $13,88 \pm 0,99$ & $9,89 \pm 1,45$ & 6,517 & 0,000 \\
Exergame & $161,13 \pm 10,65$ & $131,22 \pm 18,73$ & 3,971 & 0,001 \\
\hline
\end{tabular}

Tabela 3 - Comparação entre os escores das variáveis analisadas entre os grupos treinado e não treinado para o sexo feminino.

\begin{tabular}{lcccc}
\hline Variável & $\begin{array}{c}\text { Mulheres Treinadas } \\
\text { Média } \pm \text { DP }\end{array}$ & $\begin{array}{c}\text { Mulheres não treinadas } \\
\text { Média } \pm \text { DP }\end{array}$ & $\boldsymbol{t}$ & Sig. \\
\hline Flexibilidade & $30,48 \pm 6,35$ & $22,03 \pm 5,66$ & 2,901 & 0,011 \\
Equilíbrio & $53,00 \pm 1,85$ & $52,33 \pm 2,29$ & 0,654 & 0,523 \\
Força de membros inferiores & $14,50 \pm 1,77$ & $10,78 \pm 1,09$ & 5,281 & 0,000 \\
Exergame & $160,63 \pm 9,44$ & $128,11 \pm 10,65$ & 6,619 & 0,000 \\
\hline
\end{tabular}

Na Tabela 3 é possível observar que as mulheres do grupo treinado superam o grupo não treinado de forma significativa nos testes motores de flexibilidade e força de membros inferiores, além do escore no Exergame. No entanto, não há diferença significativa entre os níveis de equilíbrio encontrados entre os grupos.

Tabela 4 - Correlação entre os escores obtidos no Exergame com os resultados nos testes motores.

\begin{tabular}{llcc}
\hline Sexo & Variável & \multicolumn{2}{c}{ Exergame } \\
& & Sig. & r \\
\hline Masculino & Flexibilidade & 0,000 & 0,802 \\
& Equilíbrio & 0,158 & 0,358 \\
& Força de membros inferiores & 0,000 & 0,833 \\
\hline \multirow{2}{*}{ Feminino } & Flexibilidade & 0,006 & 0,641 \\
& Equilíbrio & 0,638 & 0,123 \\
& Força de membros inferiores & 0,000 & 0,898 \\
\hline
\end{tabular}

$\mathrm{Na}$ tabela 4, é possível verificar correlações estatisticamente significativas entre os escores do Exergame e os testes motores de flexibilidade e força de membros inferiores em ambos os sexos. Todas as correlações significativas são consideradas como sendo fortes.

\section{DISCUSSÃO}

O desempenho motor é parte fundamental para uma melhor qualidade de vida da população idosa. Em vista disso, torna-se essencial avaliar as qualidades físicas relacionadas ao desempenho motor da população idosa, como a flexibilidade, força de membros inferiores e equilíbrio. Atualmente, existem métodos tradicionais para mensuração e avaliação destas variáveis, sendo conhecidos como testes motores. A busca por novos protocolos eficazes para avaliar o desempenho motor de idosos se faz necessária, desde que seus resultados estejam de acordo com os encontrados nas ferramentas de avaliação já certificadas no meio científico.

Nesse sentido, estudos demonstram a eficácia da prática de exercícios físicos regulares para obtenção de melhores níveis de flexibilidade em pessoas idosas, ${ }^{20-22}$ o que justifica a diferença entre idosos treinados e não treinados, no presente estudo. A falta de exercícios físicos regulares na terceira idade traz consequências fisiológicas em relação a esse componente físico, como o encurtamento muscular. ${ }^{22}$ Estudos ressaltam que os exercícios que visam a flexibilidade podem ser encarados como grandes aliados na prevenção de futuras lesões na população idosa. ${ }^{20,24}$

Ao utilizarem o teste de sentar e alcançar Block et al. ${ }^{25}$ e Ribeiro et al. ${ }^{26}$ descrevem a facilidade estrutural e prática no uso deste instrumento, bem como sua simplicidade de aplicação para a população idosa. Ao comparar idosos praticantes e não praticantes de atividade física, Guadagnine e Olivoto, ${ }^{20}$ identificaram resultados semelhantes ao do presente estudo, em que idosos praticantes de atividade física apresentaram médias de flexibilidade significativamente maiores do que idosos não praticantes. Também Moura, ${ }^{22}$ ao comparar idosos participantes de atividades de hidroginástica e idosos não ativos fisicamente, identificaram médias no teste de sentar e alcançar de 30,37 $\pm 9,01 \mathrm{~cm}$ para idosos participantes de hidroginástica e 17,75 \pm 9,04 $\mathrm{cm}$ para idosos não praticantes.

Quanto aos resultados nos testes de força de membros inferiores dos sujeitos analisados, parece haver influência positiva da prática regular de exercícios físicos. Semelhantes aos resultados do presente estudo, Silva et al. ${ }^{27}$ identificaram valores de média da força de membros inferiores em idosos ativos $(16,1 \pm 2,8$ repetições) maiores do que em idosos não praticantes de atividades físicas $(12,1 \pm 2,7$ repetições). Também Santos et al., ${ }^{28}$ ao avaliarem a força de membros inferiores de idosos independentemente funcionais nas atividades de vida diária $(12,07 \pm 4,47$ repetições $)$ e dependentes funcionais (7,79 \pm 5,27 repetições), demonstraram a importância da prática regular de exercícios físicos, para manutenção desta variável. Para Matsudo ${ }^{19}$ a utilização do teste de Levantar da Cadeira em 30 segundos na população idosa é simples e de fácil aplicação, por se tratar de um processo prático do ponto de vista estrutural.

Em relação aos resultados obtidos no teste de equilíbrio, não foram identificadas diferenças estatisticamente significativas entre os grupos e sexo. Porém, os grupos que obtiveram melhores médias em relação 
ao equilíbrio foram os fisicamente ativos, o que pode ser justificada pela prática periódica de atividades físicas. ${ }^{28}$ Pimentel e Schecer ${ }^{29}$ demostraram também melhores médias de equilíbrio em idosos praticantes de atividades físicas $(53,6 \pm 3,7)$, quando comparados a idosos sedentários $(47,7 \pm 5,6)$. Embora a utilização da Escala de Equilíbrio de Berg seja largamente utilizada no meio científico, ${ }^{30-32}$ diferenças entre a capacidade motora da amostra investigada e da população utilizada, no estudo de Myiamoto et al., ${ }^{17}$ para a adaptação da Escala no Brasil, pode ter sido determinante para os resultados não demonstrarem diferenças entre os grupos no presente estudo. Ou seja, mesmo os idosos não treinados apresentavam uma condição física melhor que a população de pacientes do referido estudo, gerando pouca sensibilidade para encontrar diferenças entre os grupos de idosos treinados e não treinados.

Os resultados obtidos através do exergame, demostraram que os grupos treinados apresentaram melhores resultados (Sexo feminino $=160,33 \pm 9,44$; Sexo masculino $=161,13 \pm 10,65)$, quando comparados aos grupos não treinados (Sexo feminino $=128,11$ $\pm 10,65$; Sexo masculino $=131,22 \pm 18,73$ ). Segundo Sato et al., ${ }^{33}$ a utilização dos exergames podem melhorar e manter as variáveis físicas de sujeitos idosos. Pesquisadores como Agmon et al. ${ }^{34}$ e Skjaeret et al., ${ }^{14}$ tem utilizado os exergames na elaboração de treinos físicos para idosos e montagem de protocolos de atividades físicas para esta população, além de trabalharem com possibilidades de remontagem de software dos exergames para adaptação à laboratórios e clínicas específicas relacionadas à área da saúde.

No presente estudo, também ficou evidenciado a forte correlação entre os resultados do exergame e as variáveis força de membros inferiores e flexibilidade. Estudos mais recentes sugerem a utilização dos exergames na elaboração de protocolos de treinamento de curto, médio e longo prazo, sendo que resultados satisfatórios em relação à melhora do desempenho motor da população idosa já foram identificados..$^{13,35}$

A principal limitação do presente estudo é o tamanho da amostra que pode interferir na força dos resultados obtidos.

\section{CONSIDERAÇÕES FINAIS}

De acordo com os resultados obtidos, é possível concluir que o exergame pode ser utilizado como ferramenta para avaliação do desempenho motor em idosos em relação à flexibilidade e força de membros inferiores, já que seus resultados se correlacionaram significativamente com os obtidos através dos testes tradicionais para mensuração destas variáveis.

Por outro lado, o teste de equilíbrio exibido no exergame não se correlaciona com os resultados obtidos no teste tradicional desta variável. Portanto, a utilização do exergame como ferramenta para mensuração do equilíbrio parece não ser significativamente confiável. Necessita-se, portanto, de um estudo mais amplo, utilizando outras ferramentas clássicas de avaliação do equilíbrio e outras formas de utilização dos exergames nesta questão.

Por fim, cabe ressaltar que a literatura científica atual não disserta sobre a utilização dos exergames para avaliação do desempenho motor, e sim apenas para a manutenção de suas variáveis. Portanto, espera-se que os resultados obtidos neste estudo, sirvam de base para a busca de novos e alternativos métodos e ferramentas, para avaliação do desempenho motor de idosos.

Para futuros estudos sugere-se o tratamento de validação do exergame enquanto instrumento de avaliação motora, colocando-o à prova dos critérios de fidedignidade, validade e objetividade para tal validação.

\section{REFERÊNCIAS}

1. Davini R, Nunes CV. Alterações no sistema neuromuscular decorrentes do envelhecimento e o papel do exercício físico na manutenção da força muscular em indivíduos idosos. Rev Bras Fisioter 2003;7(3):201-7.

2. Schimitz VTB. Efeitos do exercício físico sobre a funcionalidade de idosos com demência: um estudo de revisão bibliográfica. Trabalho de conclusão de graduação. Porto Alegre: UFRGS, 2011.

3. Matsudo SM. Envelhecimento, atividade física e saúde. BIS, Bol. Inst. Saúde 2009;47:76-9.

4. Krug RR, Conceição JCR, Garcia GS, Streit IA, Mazo GZ. Idosos praticantes de atividades físicas: relação entre gênero e idade. Revista Biomotriz 2011;5(1):1-16.

5. Civinski C, Montibeller A, Braz AL. A Importância do Exercício Físico no Envelhecimento. Revista da Unifebe 2011;9:163-75.

6. Sousa $\mathrm{FH}$. Uma revisão bibliográfica sobre a utilização do Nintendo ${ }^{\circledR}$ Wii como instrumento terapêutico e seus fatores de risco. Revista Espaço Acadêmico 2011;11(123):155-60.

7. Ponte JM. Efeitos de um programa de cinesioterapia por meio de interação virtual em mulheres idosas. Dissertação de Mestrado. Universidade de Passo Fundo (UPF), 2013.

8. Raiter GL. Exercício físico convencional $x$ exercício físico praticado mediante consoles de videogame: efeitos nos aspectos morfólogicos, neuromusculares e fisiológicos. Trabalho de conclusão de graduação. Santa Rosa: UNIJUI, 2013.

9. Dos Santos FF, Magalhães LHVN, Sousa FAN, Marques CO, Torres MV, Leal SS. Análise da intervenção com realidade virtual e treino funcional na aptidão física de idosas em Teresina/PI. Neurociências 2015;11(2):65-71.

10. Meneghini V, Barbosa AR, Mello ALSF, Bonetti A, Guimarães AV. Percepção de adultos mais velhos quanto à participação em programa de exercício físico com exergames: estudo qualitativo. Ciênc Saúde Coletiva 2016;21(4):1033-41. doi: 10.1590/1413-81232015214.11812015

11. Vieira KL, Vaghetti CAO, Mazza SEl, Corrêa LQ. Características comportamentais de escolares e sua percepção sobre a utilização dos exergames nas aulas de Educação Física. Cinergis 2014;15(2):65-60. doi: 10.17058/ cinergis.v15i2.4431

12. Schoene D, Lord SR, Delbaere K, Severino C, Davies TA, Smith ST. A randomized controlled pilot study of home-based step training in older people using videogame technology. PLoS One 2013;8(3):e57734. doi: 10.1371/journal.pone.0057734

13. $\mathrm{Yu} \mathrm{J}, \mathrm{Kim} \mathrm{J}$. Effects of a physical activity program using exergame with elderly women. J Korean Acad Nurs 2015;45(1):84-96. doi: 10.4040/jkan.2015.45.1.84

14. Skjæret N, Nawaz A, Ystmark K, Dahl Y, Helbostad JL, Svanæs D, Vereijken B. Designing for movement quality in exergames: lessons learned from observing senior citizens playing stepping games. Gerentology 2015;61(2):186-94. 
doi: 10.1159/000365755

15. Klompstra L, Jaarsma T, Strömberg A. Exergaming to increase the exercise capacity and daily physical activity in heart failure patients: a pilot study. BMC Geriatr 2014;18(14):119. doi: 10.1186/1471-2318-14-119

16. Benedetti TRB, Antunes PC, Rodriguez-Añez CR, Mazo GZ, Petroski EL. Reprodutibilidade e validade do questionário internacional de atividade física (IPAQ) em homens idosos. Rev Bras Med Esporte 2007;13(1):11-6. doi: 10.1590/S151786922007000100004

17. Myiamoto ST, Lombardi Jl, Berg KO, Ramos IR, Natour J. Braziliam version of the Berg Balance Scale. Braz J Med Biol Rev 2004;37(9):1411-21. doi: 10.1590/S0100879X2004000900017

18. Wells KF, Dillon EK. The sit and reach: a test of back and leg flexibility. Res Q Exerc Sport 1952;23:115-8.

19. Matsudo SM. Avaliação do idoso: física e funcional. Londrina: Midiograf, 2005.

20. Guadagnine P, Olivoto R. Comparativo de flexibilidade em idosos praticantes e não praticantes de atividades físicas. Lecturas: Educación física y deportes 2004;10(69):1.

21. Vale RGS, Barreto ACG, Novaes JS, Dantas EHM. Efeitos do treinamento resistido na força máxima, na flexibilidade e na autonomia funcional de mulheres idosas. Ver Bras Cineantropom Desempenho Hum 2006;8(4):52-58.

22. Moura C. Avaliação da flexibilidade de idosos praticantes e não praticantes de hidroginástica. Trabalho de conclusão de graduação. Porto Velho: UNIR, 2008.

23. Rosa AL. A flexibilidade em indivíduos idosos. Revista de Educação do IDEAU 2012;7(15):2-15.

24. Barbanti VJ. Dicionário de Educação Física e esporte Dicionário de Educação Física e esporte Dicionário de Educação Física e esporte. Barueri: Manole, 2003.

25. Block IT, Gonçalves KK, Meyer MT, Benedito Junior S, Liberali R. Análise da flexibilidade muscular em idosos. RBPFEX 2008;2(7):141-8.

26. Ribeiro CCA, Abad CCC, Barros RV, Barros Neto TL. Nível de flexibilidade obtida pelo teste de sentar e alcançar a partir de estudo realizado na Grande São Paulo. Rev Bras Cineantropom. Desempenho Hum 2010;12(6):415-21. doi:

\subsection{7/1980-0037.2010v12n6p415}

27. Silva TCL, Costa EC, Guerra RO. Resistência aeróbia e força de membros inferiores de idosos praticantes e não-praticantes de ginástica recreativa em um centro de convivência. Rev Bras Geriatr Gerontol 2011;14(3):535-42. doi: 10.1590/ S1809-98232011000300013

28. Santos RG, Tribess S, Meneguci J, Bastos LLAG, Damião $R$, Virtuoso Júnior JS. Força de membros inferiores como indicador de incapacidade funcional em idosos. Motriz: Ver Educ Fis 2013;19(3): S35-S42. doi: 10.1590/S198065742013000700006

29. Pimentel RM, Scheicher ME. Comparação do risco de queda em idosos sedentários e ativos por meio da escala de equilíbrio de Berg. Fisioter Pesqui 2009;16(1):6-10. doi: 10.1590/S1809-29502009000100002

30. Dias BB, Mota RS, Gênova TC, Tamborelli V, Pereira VV, Puccini PT. Aplicação da Escala de Equilíbrio de Berg para verificação do equilíbrio de idosos em diferentes fases do envelhecimento. RBCEH 2009;6(3):213-24. doi: 10.5335/ rbceh.2012.194

31. Taguchi CK, Santos RD. Análise dos resultados da tendência a quedas (BBS) em idosos ativos, por meio da aplicação da Berg Balance Scale. Med Reabil 2007;26(2):10-13.

32. Wang C, Hsieh C, Olson SL, Wang C, Sheu C, Liang C. Psychometric Properties of the Berg Balance Scale in a Community-dwelling Elderly Resident Population in Taiwan. J Formos Med Assoc 2006;105(12):992-1000. doi: 10.1016/ S0929-6646(09)60283-7

33. Sato K, Kuroki K, Saiki S, Nagatomi R. Improving Walking, Muscle Strength, and Balance in the Elderly with an Exergame Using Kinect: A Randomized Controlled Trial. Games Health J 2015;4(3):161-7. doi: 10.1089/g4h.2014.0057

34. Agmon M, Perry CK, Phelan E, Demiris G, Nguyen HO. A pilot study of Wii Fit exergames to improve balance in older adults. J Geriatr Phys Ther 2011;34(4):161-8. doi: 10.1519/ JPT.0b013e3182191d98

35. Zaitsu K, Nishimura Y, Matsuguma H, Higuchi S. Association Between Extraversion and Exercise Performance Among Elderly Persons Receiving a Videogame Intervention. Games Health J 2015;4(5):375-95. doi: 10.1089/g4h.2014.0119

Como citar: PELISSER, Mateus; GARLIPP, Daniel Carlos; SANT'ANNA, Marcelo Morganti. Correlação entre os resultados obtidos em testes motores e o desempenho em exergames em idosos. Cinergis, Santa Cruz do Sul, v. 18, n. 3, maio 2017. ISSN 21774005. Disponivel em: <https://online.unisc.br/seer/index.php/cinergis/article/view/8851>. Acesso em: 22 jun. 2017. doi:http:// dx.doi.org/10.17058/cinergis.v18i3.8851. 